neurological signs persist in about $2 \%$ of patients. At follow-up $10 \%$ die of a stroke in the next ten years.

Patients deemed unsuitable for surgery are often treated with anticoagulants. Most trials show some statistically significant benefit over placebo, but how clinically significant this is remains debatable. ${ }^{19}$ Their use in arterial disease has little scientific rationale and is not without hazard. It may be that such simple remedies as aspirin, one dose of which inhibits platelet aggregation for over three days, will prove equally effective. The basis of T.I.A.s surely is to be found in the genesis of the widespread disorders which cause atheroma; improvement in our presently empirical modes of treatment is likely to be based on effective and preventive methods.

${ }^{1}$ Millikan, C. H., Stroke, 1971, 2, 201.

2 Acheson, J., and Hutchinson, E. C., Quarterly fournal of Medicine 1971, 40, 15 .

${ }^{3}$ Marshall, J., Quarterly fournal of Medicine, 1964, 33, 309

McAllen, P. M., and Marshall, J., Lancet, 1973, 1, 1212.

Mrindal, A. B., and Toole, J. F., Stroke, 1974, 5, 603.

6 Wells, C. E., Archives of Neurology, 1961, 5, 490.

Fisher, C. M., Archives of Ophthalmology, 1952, 47, 167.

Marshall, J., Medicine, 1974, 31, 838.

Bauer, R. B., et al., Fournal of the American Medical Association, 1569, 208, 509.

10 Pearce, J. M. S., Gubbarry, S. S., and Walton, J. N., Lancet, 1965, 1, 6.

\section{Hypotensive Treatment for Acute Myocardial Infarction}

The prompt recognition and consequent immediate treatment of arrhythmias in coronary care units has left the main causes of death as heart failure and cardiogenic shock. These complications are related ${ }^{1}$ to the amount of cardiac muscle necrosis: large infarcts have a higher mortality than small ones. That might, perhaps, have been expected, but the effect was somewhat masked before the otherwise lethal but treatable arrhythmias had been reduced in coronary care units, chiefly by the skill of the nursing staff. If, then, some means could be found for reducing the extent of the infarction, this inight further lessen mortality-a hope which until recently appeared to be difficult or impossible to achieve.

After an acute myocardial infarction there is an ischaemic zone around the area of irreversible necrosis, and this zone may or may not survive. ${ }^{2}$ Factors which reduce myocardial oxygen consumption favour the survival of the ischaemic zone, ${ }^{3}$ and these include a lowered velocity of contraction, decreased tension in the ventricular wall, and slowing of the heart rate. Efforts to change these factors in a favourable direction might lessen the size of eventual infarction and reduce the risks of death from heart failure and shock.

A method for assessing the size of myocardial infarction has been pioneered by American workers at San Diego and St. Louis. ${ }^{4}$ They have studied the rate of rise of the enzyme creatinine phosphokinase (CPK) after myocardial infarction. By serial studies they have been able to predict the ultimate peak levels of CPK from measurements during the first seven hours after admission. They claim that the serum levels of this enzyme, particularly when the cardiac isoenzyme is analysed, indicate the extent of myocardial necrosis, and that treatment which leads to observed CPK levels being lower than those predicted from the initial values can reasonably be considered to have reduced the size of the final infarct. These authors have now found ${ }^{5}$ that in 14 previously hypertensive patients who were admitted to hospital with acute myocardial infarction, lowering of blood pressure with trimetophan (a ganglion blocking agent) resulted in CPK levels $24 \%$ less than the predicted values. The assumption is that some $24 \%$ of jeopardized myocardium was thus salvaged from infarction. Their patients were not markedly hypertensive, the mean arterial pressure was $114 \mathrm{~mm}$ falling to $88 \mathrm{~mm}$ with treatment.

Coronary blood flow to the left ventricle is largely dependent on aortic diastolic pressure, so it might seem paradoxical to treat myocardial infarction with hypotensive agents. Until now there has been considerable concern in the management of acute infarction to avoid the undue hypotensive effects of analgesics, and most centres temporarily stop or reduce hypotensive therapy in patients with known hypertension after acute infarction. To suggest, therefore, that hypotensive treatment may be beneficial is revolutionary: the findings of Shell and Sobel indicate a way in which an inroad might possibly be made on the residual mortality. The mechanism seems logical enough-the treated patients showed a reduction in the mean left atrial pressure (the left ventricular filling pressure) while they continued to maintain a satisfactory cardiac output.

This series of 14 patients is too small for any firm conclusions to be drawn about hypotensive treatment and prognosis after acute infarction. Shell and Sobel suggest that the time has come for more extensive studies of hypotensive treatment after myocardial infarction both to assess the difference between predicted and observed $\mathrm{CPK}$ values and, more important, the effects on survival and other clinical features. Clearly such studies will need very careful monitoring of blood pressure. Many patients with acute myocardial infarction have hypertension at admission, probably due to anxiety, heart failure, or pain, while later readings may often be normal. It could be dangerous to inflict hypotension on such patients. The blood pressure should be reassessed after treatment with analgesics and for heart failure where indicated, before hypotensive therapy is started. It could well be that such trials will show a reduction in mortality in hypertensive and perhaps also in normotensive patients.

\footnotetext{
1 Harnarayan, C., et al., British Heart fournal, 1970, 32, 728.

2 Cox, J. L., et al., American Heart fournal, 1968, 76, 650

3 Maroko, P. R., et al., Circulation, 1971, 43, 67.

4 Maroko, P. R., et al., Circulation, 1971, 43, 67.

5 Shell, W. E., and Sobel, B. E., New England fournal of Medicine, 1974, 291, 481 .
}

\section{Anaesthetists' Environment}

Anaesthetists may have to work long hours, sometimes in theatres with no daylight and poor ventilation, and often without adequate time for rest and food; and the need for constant vigilance and the exacting demands of major surgery in poor risk patients might be expected to be associated with an increase in morbidity and mortality in both the anaesthetist and his patient. In fact, one recent report ${ }^{1}$ has suggested that the death rate in anaesthetists was lower than that expected. Despite earlier findings there is no evidence that anaesthetists have a higher risk of death from coronary artery disease or from malignancies of the lymphoid and reticuloendothelial tissue. Unexpectedly high rates $^{2}$ of cancer and other disease of the liver and kidney may occur in anaesthetists, however, though Spence et al. ${ }^{3}$ found no increase in the overall incidence of malignant disease among British anaesthetists. The suicide rate is considerably higher than expected and is the second or third highest of the specialty groups. ${ }^{1}$ 
At present the big cause for concern is the effect on anaesthetists of the drugs they administer. Those working in small poorly ventilated theatres accumulate inhalational anaesthetic drugs in their tissues and release them slowly. ${ }^{4}$ Contamination of the atmosphere is not necessarily confined to the theatre but may also be found in areas where patients recover. ${ }^{5}$ Repeated exposure to halothane leads to induction of liver microsomal enzyme systems; and this suggests that increased breakdown to trifluoroacetic acid, trifluoroacetyl ethanolamine, and free chloride and bromide might occur in anaesthetists who are repeatedly exposed. The accumulation of bromide ions, which are known to be slowly eliminated via the kidneys and skin, might even conceivably cause impairment of thought processes.

In order to monitor his patients closely the anaesthetist relies on auditory, visual, tactile, and even olfactory stimuli. It follows that the sensory pathways, the recognition, perception, and integration of these signals, decision making, and the motor pathways must not be impaired. Lack of adequate rest periods coupled with long hours in the theatre environment may lead to an impairment of these functions, ${ }^{4}$ an effect that may be compounded by chronic exposure to inhalational agents. ${ }^{6}$ Psychometric tests carried out on volunteers after exposure to nitrous oxide with or without halothane in concentrations comparable with those measured in theatre atmospheres have shown a decrease in ability as measured by performance tests.

Long-term exposure of animals to clinical concentrations of inhalational agents is known to produce teratrogenic effects and interfere with the immune response. Nitrous oxide can influence the white count in man and can cause fetal reabsorption and intrauterine death in rats. Whether such effects can be produced by prolonged contact with contaminated theatre atmospheres is not known. However, in 1967, the report that 18 of 31 pregnancies among 303 female Russian anaesthetists terminated in spontaneous abortion provoked renewed interest in the question. In 1970 Askrog and Harvald ${ }^{7}$ reported a high rate of spontaneous abortion among anaesthetists in Denmark, and in 1972 Knill-Jones et al. ${ }^{8}$ studied 563 married women anaesthetists and found the frequency of spontaneous abortion to be higher when anaesthetists were working than when they did not work. The miscarriage rate and the incidence of congenital abnormalities and involuntary infertility were lower in a control group. A study ${ }^{2}$ just completed by the American Society of Anesthetists and the National Institute for Occupational Safety and Health has shown clearly that there is an increased risk of spontaneous abortion among women anaesthetists and that congenital birth defects have an increased incidence among children of anaesthetists of both sexes. Whether such problems are due to pollution of the theatre atmosphere with inhalational anaesthetic agents or are due to the general working conditions remain to be determined. Until this has been established pollution should be reduced by using apparatus designed either to contain the gases (closed circuit) or to vent them out of the theatre environment. As charcoal absorbents do not clear nitrous oxide their use is possibly limited to situations when agents such as halothane are used alone.

It is welcome news that the Association of Anaesthetists of Great Britain and Ireland has set up a working party with the D.H.S.S. to consider the matter.

\footnotetext{
1 Bruce, D. L., et al., Anesthesiology, 1974, 41, 71.

2 Report of an Ad Hoc Committee on the Effect of Trace Anesthetics on the Health of Operating Room Personnel, American Society of Anesthesiologists, Anesthesiology, 1974, 41, 321. 3 Spence, A. A., et al., Proceedings of the Royal Society of Medicine, 1974,
67, 989.
}

4 Gostomzyk, J. G., et al., Anaesthestist, 1973, 22, 469.

Yanagida, H., et al., Anesthesia and Analgesia, 1974, 53, 347.

Bruce, D. L., et al., Anesthesiology, 1974, 40, 453.

Askrog, V., and Harvald, B., Nordisk Medicine, 1970, 83, 498

Knill-Jones, R. P., et al., Lancet, 1972, 1, 1326.

Wilkinson, R. T., Proceedings of the Royal Society of Medicine, 1974, 67, 994.

\section{A Nit or Not a Nit?}

The number of school children with head lice is apparently still rising. ${ }^{1}$ In 1969 Wilson reported ${ }^{2}$ that in Glasgow infestation in school girls was as common as in the 1930'snearly $10 \%$ of school entrants had nits-and that in boys the incidence had increased, with about $4 \%$ of school entrants having nits compared with less than $1 \%$ in the 1930's. He thought that this increase was due to the fashion of longer hair in boys. Two years later higher rates of infestation were found in Teeside, where Coates ${ }^{3}$ estimated that about one in every eight of the children was verminous on return to school at the beginning of one autumn term. Maunder ${ }^{4}$ showed that lice obtained from an east London school were resistant to both DDT and gammexane and he subsequently reported ${ }^{5}$ that malathion was effective in controlling the infestation. This was confirmed in a study ${ }^{6}$ in Northern Ireland, but nearly one-fifth of the children were reinfested two months after treatment.

Though there has been an increase in head infestation in children in Britain, probably owing to a combination of the development of drug resistance in lice and to the changing fashions in the style and care of hair, head infestation is sometimes misdiagnosed and some of the reported increase may be spurious. Kutz ${ }^{7}$ reported an incident where an extensive infestation with "nits" proved to be a misdiagnosis, for the so-called nits were shown to be minute pieces of scalp which had become glued around the hair, probably by hair spray. In another "outbreak" small opaque globules attached to the hair were mistaken for nits. ${ }^{8}$ These episodes illustrate the importance of confirming the diagnosis of infestation by expert examination with a lens, particularly in the absence of adult lice.

Malathion lotion $(0.5 \%$ in spirit) has been shown to be effective in killing lice and nits resistant to DDT and gammexane and also to have a residual effect in the scalp. It seems now to be the treatment of choice in areas where resistance has appeared. In these areas malathion treatment should completely replace treatment with DDT and gammexane. The treatment with malathion is simple: the lotion should be applied freely to the scalp and then allowed to dry (heat should not be applied because the lotion is spirit based and therefore inflammable). The head should not be washed until about 24 hours after the application of the malathion, and then shampooing and combing may be carried out to remove the dead nits.

Head louse infestation is usually a family condition, so that treatment should not be confined to the school child if reinfestation is to be avoided. The nurse should visit the house of the affected child and explain that infestation is spread by close contact, irrespective of the state of cleanliness, and that lice do not imply a "dirty head." She should then offer malathion treatment to all members of the household, whether or not she can verify current infestation, because only by family treatment can eradication be achieved.

Resistance of lice to malathion may soon appear in Britain; Silverton ${ }^{9}$ quoted a case where malathion treatment appeared 